Quim. Nova, Vol. 29, No. 1, 66-71, 2006

\title{
AVALIAÇÃO DE CONTAMINANTES INORGÂNICOS E ORGÂNICOS EM ÁLCOOL COMBUSTÍVEL UTILIZANDO ELETROFORESE CAPILAR
}

\author{
Elisabete A. Pereira* e Marina F. M. Tavares \\ Instituto de Química, Universidade de São Paulo, CP 26077, 05513-970 São Paulo - SP \\ Alessandra Stevanato e Arnaldo A. Cardoso \\ Instituto de Química, Universidade Estadual Paulista, CP 355, 14801-970 Araraquara - SP
}

Recebido em 20/12/04; aceito em 4/5/05; publicado na web em 24/8/05

\begin{abstract}
EVALUATION OF INORGANIC AND ORGANIC CONTAMINANTS IN ALCOHOL FUEL BY CAPILLARY ELECTROPHORESIS. This work reports the analysis of inorganic and organic contaminants in alcohol fuel samples using capillary electrophoresis. Chloride and sulfate were analyzed in nitrate/ monochloroacetic acid at $10 \mathrm{mmol} \mathrm{L}^{-1}$ concentration each under indirect UV detection $(210 \mathrm{~nm})$. The analysis of aldehydes is based on the $216 \mathrm{~nm}$ detection of 3-methyl-2-benzothiazoline hydrazone adducts. The running buffer consisted of $20 \mathrm{mmol} \mathrm{L}^{-1}$ tetraborate , $40 \mathrm{mmol} \mathrm{L}^{-1}$ sodium dodecyl sufate and $12 \mathrm{mmol} \mathrm{L}^{-1} \beta$-ciclodextrin. Both methodologies were applied to real samples indicating inorganic ion concentrations from 0.15 to $6.64 \mathrm{mg} \mathrm{kg}^{-1}$ and aldehydes from 32.0 to $91.3 \mathrm{mg} \mathrm{L}^{-1}$.
\end{abstract}

Keywords: inorganic and organic contaminants; alcohol fuel; capillary electrophoresis.

\section{INTRODUÇÃO}

Ao final do século XIX o petróleo tornou-se uma fonte importante de energia. Durante a maior parte do século XX, apesar do aumento da demanda e consequiente aumento da produção, o preço do petróleo, controlado pelas grandes multinacionais petrolíferas, foi mantido estável até início da década de 70. A instabilidade política na maior região produtora, o Oriente Médio, criou uma série de conflitos que resultou na guerra do Youn Kippur. Como resultado do conflito, os países da região produtores de petróleo criaram a OPEP (Organização dos Países Exportadores de Petróleo) que passou a impor os preços e controlar a produção como forma de pressão política contra Israel e seus simpatizantes ${ }^{1}$.

A política de preços da OPEP atingiu fortemente o Brasil, já que na época a produção local de petróleo representava apenas $23 \%$ do consumo interno. Em um único ano, 1973 a 1974, as despesas com a importação de combustível aumentaram de 600 milhões para 2 bilhões de dólares. Para minimizar o efeito na balança de pagamentos entre importação e exportação e diminuir sua vulnerabilidade energética, em novembro de 1975, o governo brasileiro criou o Programa Nacional do Álcool - PROÁLCOOL, que tinha como objetivo a produção de álcool combustível ${ }^{2}$.

O resultado inicial do PROÁLCOOL foi a produção de etanol anidro usado como componente da gasolina. Posteriormente ele passou também a ser usado na forma hidratada como fonte única de combustível de motores a explosão, abrindo assim o espaço para uma nova matriz energética proveniente de uma fonte renovável. Apesar do uso do álcool ter como meta inicial a solução de um problema econômico, indiretamente algumas vantagens ambientais resultaram do uso do produto. Podemos citar como principais vantagens a eliminação do uso de aditivos à base de chumbo, já que o etanol é um combustível com alto poder de compressão; comparado à gasolina, o uso do etanol reduz as emissões de $\mathrm{SO}_{2}, \mathrm{CO}$ e

*e-mail: ealves@iq.usp.br hidrocarbonetos ${ }^{3}$ e por ser um combustível proveniente de fonte renovável e, considerando-se o processo global de plantio, produção e uso, que resulta em ciclo fechado para o elemento carbono, seu uso não contribui efetivamente para aumento do estoque de carbono atmosférico ${ }^{4}$. Atualmente, com a diminuição do poder de pressão da OPEP, é justamente o aspecto ambiental que aparece como forma efetiva e crescente pressão sobre os países desenvolvidos e deve impulsionar o uso do etanol como combustível no planeta, principalmente caso o protocolo de Kyoto venha a ser ratificado pelos países industrializados.

O crescente uso do etanol no panorama brasileiro e mundial, seja como combustível ou matéria-prima para indústrias, justifica um rígido controle da qualidade do álcool vendido, tanto no atacado como no varejo, isto porque a produção de álcool no Brasil, que é da ordem de 15 bilhões de L por safra ${ }^{5}$, é feita por inúmeras destilarias espalhadas no país, fato que dificulta a padronização do produto final.

A Agência Nacional do Petróleo (ANP) é o órgão regulador federal que estabelece os padrões do álcool combustível produzido e comercializado no país. Para cloreto e sulfato, os limites variam de acordo com o tipo de combustível, por ex., em etanol hidratado os limites são de $1 \mathrm{mg} \mathrm{kg}^{-1}$ para cloreto e $4 \mathrm{mg} \mathrm{kg}^{-1}$ para sulfato ${ }^{6}$. Para os aldeídos não há normas estabelecidas até o momento. Segundo Seinfeld e colaboradores ${ }^{7}$, o transporte, manuseio e uso de combustível é responsável pela maior parte da emissão de compostos orgânicos voláteis para a atmosfera nos grandes centros urbanos, fato este que sugere a necessidade de se conhecer a composição do álcool passível de emissão. Pelo lado da segurança ambiental, também é fundamental o conhecimento da composição do álcool para que existam parâmetros que permitam o estabelecimento de normas de estocagem e manipulação de álcool combustível.

Os métodos clássicos para análise de ânions inorgânicos são baseados em métodos por via úmida, colorimétricos e cromatográficos ${ }^{8,9}$. Já a determinação de aldeídos em álcool é baseada em medidas colorimétricas e cromatográficas ${ }^{10-12}$. Métodos por via úmida são pouco sensíveis, além de exigir diferentes etapas analíticas. Os métodos cromatográficos têm a vantagem de poder especiar 
os componentes, mas apresentam como desvantagens elevado consumo de solventes, longos tempos de análise, alto custo, entre outros. $\mathrm{O}$ método recomendado ${ }^{12}$ para aldeídos requer uma intensa purificação do reagente e dos solventes antes de serem utilizados na etapa de derivação. Dentre as técnicas de separação que poderiam servir para especiação e determinação de íons e aldeídos, a eletroforese capilar $(\mathrm{CE})^{13}$ é ainda pouco explorada para este tipo de matriz ${ }^{14}$, apesar de ser uma técnica consolidada para determinação de uma grande variedade de compostos presentes em matrizes diversas ${ }^{15,16}$. A eletroforese capilar apresenta vantagens como rapidez, eficiência, menor custo por análise, além do reduzido consumo de reagentes, um diferencial sob o aspecto ambiental já que minimiza a geração de resíduo analítico.

O presente trabalho apresenta métodos alternativos utilizando os modos de eletroforese capilar em solução livre (FSCE) e cromatografia eletrocinética micelar (MEKC) para determinação de ânions inorgânicos e aldeídos, respectivamente, em amostras de álcool combustível.

\section{PARTE EXPERIMENTAL}

\section{Reagentes e soluções}

Todos os reagentes e solventes utilizados foram de grau analítico. Os sais de sulfato de sódio, cloreto de sódio, nitrato de sódio, formaldeído, acetaldeído, acetona, $\beta$-ciclodextrina, tetraborato de sódio, 2,4-dinitrofenil-hidrazina (DNPH) e 3-metil-2-benzotiazolinona hidrazona (MBTH) foram obtidos da Aldrich/Sigma (St. Louis, MO, USA). Dodecil sulfato de sódio (SDS) foi obtido da Riedel Haen (Seelze, Germany).

As soluções estoque padrão de cloreto e sulfato foram preparadas pela dissolução de uma quantidade apropriada dos sais de sódio em etanol absoluto e estocadas sob refrigeração. As concentrações preparadas foram de $65,36 \mathrm{mg} \mathrm{kg}^{-1}$ para cloreto e $68,67 \mathrm{mg} \mathrm{kg}^{-1}$ para sulfato.

Solução estoque nitrato de sódio foi preparada na concentração de $50 \mathrm{mmol} \mathrm{L}^{-1}$. Soluções estoques de SDS e tetraborato foram preparadas na concentração de $100 \mathrm{mmol} \mathrm{L}^{-1}$.

Soluções estoque padrão dos aldeídos $\left(1000 \mathrm{mg} \mathrm{L}^{-1}\right)$ foram preparadas pela dissolução de quantidades apropriadas dos aldeídos em água deionizada (Milli-Q purification system, Millipore Corp., Bedford, MA, USA). A reação de derivação foi feita misturando-se quantidades apropriadas de formaldeído, acetaldeído e acetona com $2 \mathrm{~mL}$ de MBTH $(0,1 \%)$. Esta mistura permaneceu em repouso por $1 \mathrm{~h}$. Em seguida, adicionou-se $1,5 \mathrm{~mL}$ de tetraborato de sódio $\left(100 \mathrm{mmol} \mathrm{L}^{-1}\right)$ à mistura e o volume final de $5,0 \mathrm{ml}$ foi ajustado com água deionizada.

Etanol livre de compostos carbonílicos foi obtido por destilação de etanol em presença de DNPH (1 L de solvente, 0,5 g DNPH e 10 gotas de ácido sulfúrico). O solvente purificado foi recolhido em um balão de $0,5 \mathrm{~L}$ quando a temperatura de destilação encontrava-se em torno de $78^{\circ} \mathrm{C}^{17}$.

O condicionamento do capilar foi feito através da passagem de solução de hidróxido de sódio $1,0 \mathrm{~mol} \mathrm{~L}^{-1}$ durante $5 \mathrm{~min}$, seguida de água deionizada durante 5 min e solução de eletrólito (40 min), no início de cada dia ou quando foi efetuada mudança de condição de análise. Entre as corridas o capilar foi condicionado com eletrólito durante $3 \mathrm{~min}$.

\section{Padrões e amostras}

As soluções padrão de trabalho de cloreto e sulfato foram preparadas por diluição da solução estoque e ajuste do volume para $5,0 \mathrm{~mL}$ com etanol absoluto. A solução padrão foi evaporada
(60 ${ }^{\circ} \mathrm{C}$ em fluxo de nitrogênio) e o resíduo dissolvido em $500 \mu \mathrm{L}$ de água deionizada para posteriormente ser determinada por CE.

As amostras de álcool combustível foram coletadas em postos de gasolina locais. Alíquotas de $5 \mathrm{~mL}$ de álcool combustível foram precisamente pesadas. As amostras foram tratadas por evaporação ( $60{ }^{\circ} \mathrm{C}$ em fluxo de nitrogênio). O resíduo foi dissolvido em $500 \mu \mathrm{L}$ de água deionizada para posteriormente ser analisado por CE. O procedimento de evaporação/redissolução teve como objetivo eliminar o solvente orgânico e, em paralelo, concentrar em 10 vezes os analitos na nova solução aquosa.

As soluções padrão de formaldeído, acetaldeído e acetona foram preparadas por diluição da solução estoque em etanol purificado.

Para as determinações dos contaminantes orgânicos, uma alíquota de 1,0 mL de álcool combustível foi misturada com $2 \mathrm{~mL}$ de $0,1 \%$ de MBTH. Após $1 \mathrm{~h}$ de reação adicionou-se $1,5 \mathrm{~mL}$ de tetraborato de sódio $\left(100 \mathrm{mmol} \mathrm{L}^{-1}\right)$ e o volume final $(5,0 \mathrm{~mL})$ foi ajustado com água deionizada.

\section{Recuperação}

Para determinar a exatidão dos métodos propostos, ensaios de recuperação foram realizados de acordo com os protocolos descritos na literatura ${ }^{18}$.

Os testes de recuperação para os ânions inorgânicos foram realizados fortificando-se a matriz (etanol absoluto) com cloreto e sulfato, em três diferentes níveis de concentração. A porcentagem de recuperação foi determinada pela comparação de determinações feitas em triplicata das soluções padrão submetidas ao procedimento de evaporação, com soluções padrão não-submetidas ao procedimento, as quais representam $100 \%$ de recuperação.

Para os ensaios de recuperação dos contaminantes orgânicos, amostras de álcool combustível foram fortificadas com acetaldeído e acetona em três diferentes níveis de concentração. A porcentagem de recuperação foi calculada de acordo com o procedimento descrito pela AOAC International ${ }^{19}$.

\section{Instrumentação}

Todos os experimentos foram realizados no equipamento modelo HP 3D CE da Agilent, equipado com fonte de alta tensão $(0-30 \mathrm{kV})$, detector de arranjo de diodos, que opera na faixa de $190-600 \mathrm{~nm}$, controlador de temperatura e com programa de aquisição e tratamento de dados (ChemStation, USA). Os capilares de sílica fundida utilizados são da Polymicro Technologies, Phoenix, AZ, com dimensões de $75 \mu \mathrm{m}$ d. i. e 48,5 $\mathrm{cm}$ de comprimento total (40 cm até o detector). Uma janela de detecção de aproximadamente $0,3 \mathrm{~cm}$ foi aberta pela remoção do revestimento de poliimida do capilar por aquecimento.

Os ânions inorgânicos foram injetados pelo modo hidrodinâmico com pressão $5000 \mathrm{~Pa}$ x $10 \mathrm{~s}$ e detectados indiretamente em $210 \mathrm{~nm}$. Os adutos analito-MBTH foram injetados pelo modo hidrodinâmico com pressão $2500 \mathrm{~Pa}$ x $5 \mathrm{~s}$ e detectados diretamente em $216 \mathrm{~nm}$.

\section{RESULTADOS E DISCUSSÃO}

\section{Ânions inorgânicos}

Tendo em vista que a maioria dos íons inorgânicos possuem baixa ou nenhuma absorção na região do UV, a detecção indireta é o modo de detecção mais utilizado para análise de ânions por CE. Neste tipo de detecção, o eletrólito deve conter um cromóforo com alta absortividade molar, de modo que na passagem do soluto pelo detector exista um deslocamento do cromóforo e queda do sinal analítico $^{20}$. O nitrato foi selecionado por preencher os principais 
requisitos de um eletrólito de corrida: cromóforo, mobilidade eletroforética próxima aos ânions analisados e estabilidade elevada em solução.

A maioria dos métodos existentes na literatura utilizando a $\mathrm{CE}$ para análise de ânions inorgânicos descrevem separações conduzidas sob inversão de fluxo ${ }^{21,22}$. Sob esta condição, um sal quaternário de amônio deve ser adicionado ao eletrólito para inverter a direção do fluxo eletrosmótico (EOF). Entretanto, em meio suficientemente ácido $(\mathrm{pH}<3)$, o EOF, em capilares de sílica fundida, é praticamente eliminado, e os ânions podem ser separados simplesmente por diferença entre suas mobilidades eletroforéticas. Thornton e Fritz $^{23}$ demostraram que excelentes separações de ânions são possíveis de serem realizadas em meio ácido.

Para desenvolvimento do método, inicialmente utilizou-se um eletrólito de corrida contendo de $5 \mathrm{mmol} \mathrm{L}^{-1}$ de nitrato de sódio e $10 \mathrm{mmol} \mathrm{L}^{-1}$ de ácido monocloroacético, $\mathrm{pH} 2$. O eletrólito selecionado proporcionou boa separação dos ânions investigados (cloreto e sulfato), mas apresentou instabilidade na linha de base (resultados não apresentados).

A influência da concentração do nitrato, no sistema de separação, foi investigada no intervalo de 5-15 $\mathrm{mmol} \mathrm{L}^{-1}$, mantendo constantes a concentração de ácido monocloroacético, o pH e a tensão aplicada (10 mmol L-1 de ácido monocloroacético, $\mathrm{pH} 2,-12 \mathrm{kV}$ ). $\mathrm{O}$ aumento da concentração de nitrato estabilizou a linha de base e proporcionou um aumento significativo do sinal analítico para o íon cloreto. De acordo com a literatura ${ }^{24}$, oscilações na linha de base e imprecisão nas medidas de mobilidade podem ser observadas quando eletrólitos com baixa concentração são utilizados. Sob esta condição, a eletrosmose torna-se muito sensível à presença de impurezas, mudanças no $\mathrm{pH}$, etc. $\mathrm{O}$ aumento do sinal pode ser simplesmente devido ao aumento da concentração de nitrato, o ânion cromóforo do eletrólito, ou estar relacionado com a diferença de força iônica entre a amostra e o eletrólito, efeito conhecido como "stacking" 25 . Por outro lado, a desvantagem de utilizar eletrólitos altamente concentrados é o aumento do calor gerado no capilar quando a tensão é aplicada (efeito Joule) ${ }^{26}$. A concentração de $10 \mathrm{mmol} \mathrm{L}^{-1}$ de nitrato foi selecionada por apresentar uma boa relação entre a magnitude do sinal analítico e a corrente gerada.

Outro parâmetro avaliado foi o tipo de injeção da amostra. Em $\mathrm{CE}$, as amostras podem ser injetadas no capilar por métodos hidrodinâmicos (HD) ou eletrocinéticos (EK). Na injeção hidrodinâmica utiliza-se um gradiente de pressão. Na injeção eletrocinética, um gradiente de potencial é estabelecido ao longo do comprimento do capilar por um período de tempo conhecido ${ }^{27}$. A Tabela 1 apresenta os resultados obtidos. O modo de injeção hidrodinâmico mostrou-se superior, em relação à repetibilidade, quando comparado ao modo eletrocinético. A Figura 1 apresenta a separação de cloreto e sulfato sob condições otimizadas.

A precisão do método, utilizando o modo de injeção $\mathrm{HD}$, foi determinada em relação ao tempo de migração e área dos picos. Os

Tabela 1. Comparação entre os modos de injeção HD e EK para análise de cloreto e sulfato

\begin{tabular}{lcccc}
\hline & $\begin{array}{c}\text { Tempo de migração } \\
\% \mathrm{CV}^{*}\end{array}$ & \multicolumn{3}{c}{$\begin{array}{c}\text { Área do pico } \\
\% \mathrm{CV}^{*}\end{array}$} \\
\hline ânion & modo HD & modo EK & modo HD & modo EK \\
\hline Cloreto & 0,45 & 0,58 & 2,4 & 4,2 \\
Sulfato & 0,46 & 0,66 & 1,1 & 4,1 \\
\hline
\end{tabular}

HD = injeção hidrodinâmica (10 s x $5000 \mathrm{~Pa})$; EK = injecão eletrocinética $(5 \mathrm{~s} \mathrm{x}-2,5 \mathrm{kV}) ;{ }^{*} \mathrm{CV}=$ Coeficiente de variação $(\mathrm{n}=10)$

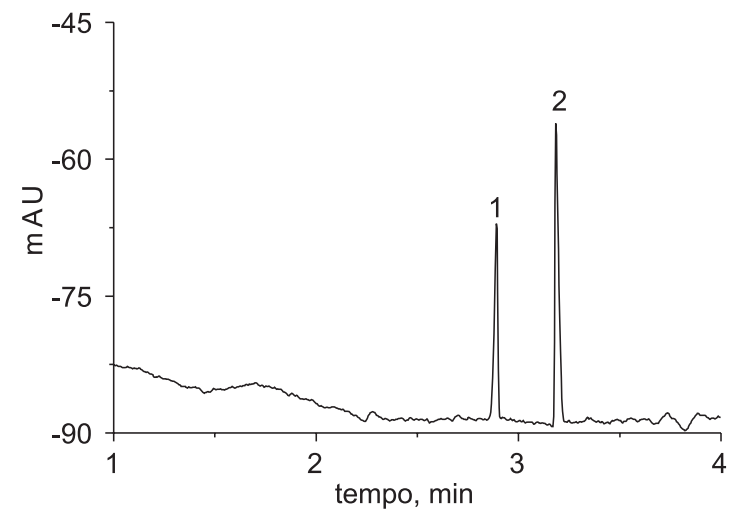

Figura 1. Condições otimizadas para análise de ânions inorgânicos. Concentração de cada ânion: $2,5 \mathrm{mg} \mathrm{kg}^{-1}$ de cloreto e 5,0 $\mathrm{mg} \mathrm{kg}^{-1}$ de sulfato. Capilar de $75 \mu \mathrm{m} \times 48,5 \mathrm{~cm}$ (40 cm até o detector). Eletrólito: $10 \mathrm{mmol} \mathrm{L}^{-1}$ nitrato de sódio e $10 \mathrm{mmol} \mathrm{L}^{-1}$ ácido monocloroacético, pH 2; injeção: 10 s $x$ 5000 Pa; tensão: - $12 \mathrm{kV}$; temperatura: $25^{\circ} \mathrm{C}$; detecção indireta: $210 \mathrm{~nm} .1$. Cloreto e 2. Sulfato

valores de coeficiente de variação foram inferiores a $0,50 \%$ para tempo de migração e 2,5\% para área dos picos (Tabela 1).

A exatidão do método proposto foi calculada como a porcentagem de recuperação de uma quantidade conhecida de analito adicionada à matriz (etanol absoluto). Os resultados estão apresentados na Tabela 2. Os testes de recuperação mostraram valores que variaram de 85 a $103 \%$ para os níveis de concentração estudados. Estes resultados indicam boa exatidão, de acordo com o protocolo de validação do AOAC que estabelece valores de recuperação no intervalo de 80 a $110 \%{ }^{28}$.

Tabela 2. Ensaios de recuperação

\begin{tabular}{lccc}
\hline Ânion & $\begin{array}{c}\text { Concentração } \\
\text { adicionada } \\
\mathrm{mg} \mathrm{kg}^{-1}\end{array}$ & $\begin{array}{c}\text { Concentração } \\
\text { encontrada } \\
\mathrm{mg} \mathrm{kg}^{-1}\end{array}$ & $\begin{array}{c}\text { Recuperação } \\
\%\end{array}$ \\
\hline Cloreto & 2,06 & 2,10 & $102 \pm 2$ \\
Sulfato & 2,18 & 1,86 & $85,4 \pm 1,5$ \\
Cloreto & 4,14 & 4,28 & $103 \pm 2$ \\
Sulfato & 4,34 & 4,22 & $97,1 \pm 1,5$ \\
Cloreto & 8,28 & 7,81 & $94,3 \pm 1,8$ \\
Sulfato & 8,69 & 8,51 & $97,9 \pm 1,8$ \\
\hline
\end{tabular}

Para estabelecer a linearidade do método, alíquotas da matriz foram fortificadas com padrões de cloreto e sulfato em cinco diferentes níveis de concentração. Os parâmetros estatísticos apresentados na Tabela 3 mostram que, as curvas de calibração apresentaram boa linearidade $(r>0,996)$ na faixa de concentração de $0,02-0,1 \mathrm{mg} \mathrm{kg}^{-1}$ para cloreto e $0,20-4,0 \mathrm{mg} \mathrm{kg}^{-1}$ para sulfato. Cabe mencionar que os limites de detecção instrumentais para cloreto e sulfato foram 0,16 e $0,27 \mathrm{mg} \mathrm{kg}^{-1}$, respectivamente. Desde que cada padrão foi pré-concentrado por um fator de dez durante a preparação, os LODs do método são cerca de 10 vezes menores que os LODs instrumentais.

Amostras de álcool combustível foram analisadas pelo método proposto. Os resultados obtidos estão apresentados na Figura 2 e Tabela 4. As amostras 01 e 04 apresentaram concentrações de sulfato acima do valor estabelecido pela ANP. Todas as amostras apresentaram, com exceção da amostra 02 , concentrações de cloreto abaixo do limite de quantificação e, conseqüentemente, abaixo do valor estabelecido pela ANP. 
Tabela 3. Validação do método em termos de linearidade, limites de detecção (LOD) e quantificação (LOQ)

\begin{tabular}{lccccc}
\hline Ânion & curva de calibração & Coeficiente de correlação & LOD c/mg kg-1 & LOQ $^{\text {d } / \mathrm{mg} \mathrm{kg}^{-1}}$ & SD \\
\hline Cloreto $^{\text {a }}$ & $\mathrm{Y}=9,82 \mathrm{X}+0,034$ & 0,9964 & 0,016 & 0,048 & 0,04772 \\
Sulfato $^{\mathrm{b}}$ & $\mathrm{Y}=1,42 \mathrm{X}+0,006$ & 0,9976 & 0,027 & 0,080 & 0,01143 \\
\hline
\end{tabular}

${ }^{a}$ Curva de calibração no intervalo de $0,02-0,1 \mathrm{mg} \mathrm{kg}^{-1}$; ${ }^{\mathrm{b}}$ curva de calibração no intervalo de $0,20-4,0 \mathrm{mg} \mathrm{kg}^{-1} ;{ }^{\mathrm{c}}$ calculado de acordo com a literatura ${ }^{31} \mathrm{LOD}=3,3 \mathrm{SD} / \mathrm{B} ;{ }^{\mathrm{d}} \mathrm{LOQ}=10 \mathrm{SD} / \mathrm{B}$, onde $\mathrm{SD}$ é o desvio padrão da resposta e $\mathrm{B}$ é a inclinação da curva de calibração.

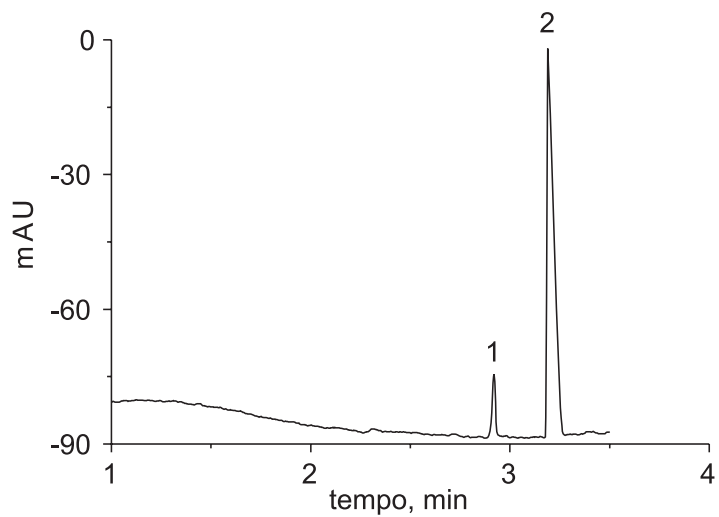

Figura 2. Eletroferograma de uma amostra de álcool combustível para inspeção de ânions. Condições experimentais e legenda como descrito na Figura 1

Tabela 4. Resultado das análises de cloreto e sulfato em amostras de álcool combustível

\begin{tabular}{cc}
\hline \multicolumn{1}{c|}{ Ânion } & $\begin{array}{c}\text { Concentração } \\
\mathrm{mg} \mathrm{kg}^{-1}\end{array}$ \\
\hline Amostra 01 & $<$ LOQ \\
Cloreto & $5,73 \pm 0,07$ \\
Sulfato & $0,150 \pm 0,004$ \\
Amostra 02 & $3,60 \pm 0,04$ \\
Cloreto & $<$ LOQ \\
Sulfato & $3,54 \pm 0,05$ \\
Amostra 03 & $<$ LOQ \\
Cloreto & $6,64 \pm 0,08$ \\
Sulfato & \\
Amostra 04 & \\
Cloreto & Sulfato
\end{tabular}

\# duplicata

\section{Contaminantes orgânicos}

A análise de aldeídos alifáticos por $\mathrm{CE}$ apresenta dois desafios: os aldeídos são compostos que não apresentam carga e não absorvem na região UV (200 - $360 \mathrm{~nm})$. Essa limitação pode ser superada utilizando-se a derivação. Os aldeídos e a acetona reagem com MBTH para formar um aduto neutro, com máximo de absorção em $216 \mathrm{~nm}^{12}$. O MBTH apresenta como vantagens alta solubilidade em solventes polares e não requer prévia purificação.

Para os experimentos preliminares utilizou-se uma mistura padrão contendo $10 \mathrm{mg} \mathrm{L}^{-1}$ de cada aldeído e acetona derivatizados com $0,1 \%$ de MBTH (como descrito na seção experimental). Os parâmetros instrumentais e analíticos foram selecionados com base na literatura ${ }^{29}$. A MEKC é usualmente feita com um eletrólito de corrida, que contém somente um tampão e um tensoativo. Outras substâncias, denominadas modificadores, podem ser adicionadas ao eletrólito de corrida com o objetivo de alterar a seletividade do meio. O efeito da adição de modificadores ao eletrólito de corrida na separação dos analitos-MBTH foi investigado. A adição de $\beta$-ciclodextrina $(\beta-C D)$ ao eletrólito não proporcionou variações significativas no sinal analítico, quando comparado com os resultados obtidos ao utilizar-se como eletrólito $50 \mathrm{mmol} \mathrm{L}^{-1}$ de SDS e $20 \mathrm{mmol} \mathrm{L}^{-1}$ de tetraborato de sódio, pH 9 (resultados não apresentados). Entretanto a $\beta$-CD promoveu o desdobramento do pico do acetaldeído, freqüentemente atribuído aos isômeros cis-trans formados durante o processo de derivação ${ }^{30}$. Com base nos resultados obtidos optou-se pelo uso do eletrólito contendo $\beta$-ciclodextrina (Figura 3). A concentração de SDS, inicialmente estabelecida em $50 \mathrm{mmol} \mathrm{L}^{-1}$, foi reduzida para $40 \mathrm{mmol} \mathrm{L}^{-1}$ com o objetivo de diminuir o tempo de análise e o aquecimento gerado no capilar.

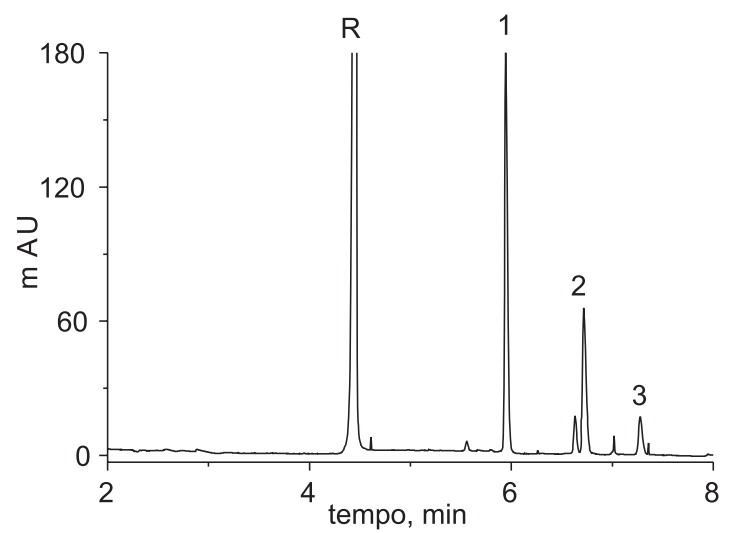

Figura 3. Separação da mistura padrão de aldeídos e acetona $\left(10 \mathrm{mg} \mathrm{L}^{-1}\right.$ de cada) derivatizados com 0,1\% de MBTH. Capilar de sílica fundida $75 \mu m \times 48,5$ $\mathrm{cm}$. Eletrólito: $50 \mathrm{mmol}^{-1}$ de SDS contendo $20 \mathrm{mmol} \mathrm{L}^{-1}$ de tetraborato de sódio e $12 \mathrm{mmol} \mathrm{L}^{-1} \mathrm{de} \beta$-CD, 5 sx $2500 \mathrm{~Pa}$; tensão: $20 \mathrm{kV}$; temperatura: $27^{\circ} \mathrm{C}$; detecção em 216 nm. R. Reagente, 1. Formaldeído, 2. Acetaldeído, 3. Acetona

O método foi validado segundo os parâmetros de linearidade, precisão, recuperação, limite de detecção e quantificação.

A repetibilidade do método em termos de tempo de migração e área do pico foi determinada quanto ao coeficiente de variação para dez injeções consecutivas da mistura padrão contendo $10 \mathrm{mg} \mathrm{L}^{-1}$ de cada analito de interesse. A Tabela 5 apresenta os resultados obtidos. Os coeficientes de variação foram inferiores a 1,0\% para tempo de migração e 2,0\% para área dos picos.

Tabela 5. Desvio padrão relativo dos tempos de migração, área e altura dos picos dos analitos-MBTH

\begin{tabular}{lccc}
\hline Analito-MBTH & $\begin{array}{c}\text { Tempo de } \\
\text { migração CV\% }\end{array}$ & $\begin{array}{c}\text { Área do } \\
\text { pico CV\% }\end{array}$ & $\begin{array}{c}\text { Altura do } \\
\text { pico CV\% }\end{array}$ \\
\hline Formaldeído & 0,68 & 1,2 & 6,4 \\
Acetaldeído & 0,80 & 1,4 & 8,6 \\
Acetona & 0,91 & 1,8 & 3,9 \\
\hline
\end{tabular}

injeção hidrodinâmica (5 s x 2500 Pa ); CV = Coeficiente de variação $(\mathrm{n}=10)$ 
A Tabela 6 mostra a exatidão do método com valores de recuperação que variaram de 93,8 a 99,7\% para acetaldeído e de 93,7 a 95,8\% para acetona.

Avaliou-se a linearidade do método proposto com amostras de etanol destilado fortificadas com padrões de acetaldeído e acetona em cinco diferentes níveis de concentração. $\mathrm{O}$ método apresentou boa linearidade $(r>0,998)$ dentro da faixa de concentração estudada. Os LODs para acetaldeído e acetona foram na ordem de 0,45 $\mathrm{mg} \mathrm{L}^{-1}$ e 0,58 $\mathrm{mg} \mathrm{L}^{-1}$, respectivamente (Tabela 7).

Amostras de diferentes procedências foram analisadas pelo método proposto. A derivação foi feita como descrito na parte experimental. Os resultados obtidos estão apresentados na Tabela $8 \mathrm{e}$ Figura 4. Os resultados indicam que o acetaldeído é a impureza majoritária e sua concentração possui uma ampla variação nas diferentes amostras avaliadas.
Tabela 6. Teste de recuperação

\begin{tabular}{lccc}
\hline Analito-MBTH & $\begin{array}{c}\text { Concentração } \\
\text { adicionada } \\
\text { mg L }^{-1}\end{array}$ & $\begin{array}{c}\text { Concentração } \\
\text { encontrada } \\
\text { mg L}^{-1}\end{array}$ & $\begin{array}{c}\text { Recuperação } \\
\%\end{array}$ \\
\hline Acetaldeído & 5,00 & 4,82 & $96,4 \pm 1,6$ \\
Acetona & 5,00 & 4,79 & $95,8 \pm 1,7$ \\
Acetaldeído & 10,00 & 9,38 & $93,8 \pm 1,4$ \\
Acetona & 10,00 & 9,42 & $94,2 \pm 1,8$ \\
Acetaldeído & 20,00 & 19,94 & $99,7 \pm 1,5$ \\
Acetona & 20,00 & 18,76 & $93,8 \pm 1,8$ \\
\hline
\end{tabular}

injeção hidrodinâmica (5 s x $2500 \mathrm{~Pa}$ ); CV = Coeficiente de variação $(\mathrm{n}=10)$

Tabela 7. Validação do método em termos de linearidade, limites de detecção (LOD) e quantificação (LOQ)

\begin{tabular}{|c|c|c|c|c|c|}
\hline Analito-MBTH & Curva de calibração ${ }^{\text {a }}$ & Coeficiente de correlação & $\mathrm{LOD}^{\mathrm{b}} \mathrm{mg} \mathrm{L}^{-1}$ & $\mathrm{LOQ}^{\mathrm{c}} \mathrm{mg} \mathrm{L}^{-1}$ & SD \\
\hline Acetaldeído & $Y=18,66 X+1,95$ & 0,9996 & 0,45 & 1,35 & 2,5169 \\
\hline Acetona & $Y=0,92 X-0,97$ & 0,9987 & 0,58 & 1,76 & 0,1612 \\
\hline
\end{tabular}

${ }^{\mathrm{a}}$ Curva de calibração no intervalo de $1,0-20,0 \mathrm{mg} \mathrm{L}{ }^{-1} ;{ }^{\mathrm{b}} \mathrm{LOD}=3,3 \mathrm{SD} / \mathrm{B} ;{ }^{\mathrm{c}} \mathrm{LOQ}=10 \mathrm{SD} / \mathrm{B}$, onde SD é o desvio padrão da resposta e B é a inclinação da curva de calibração.

a)

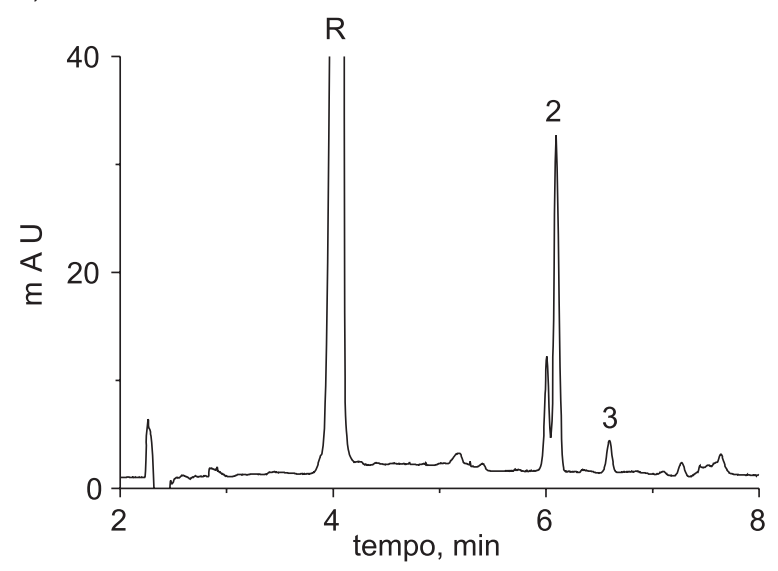

b)

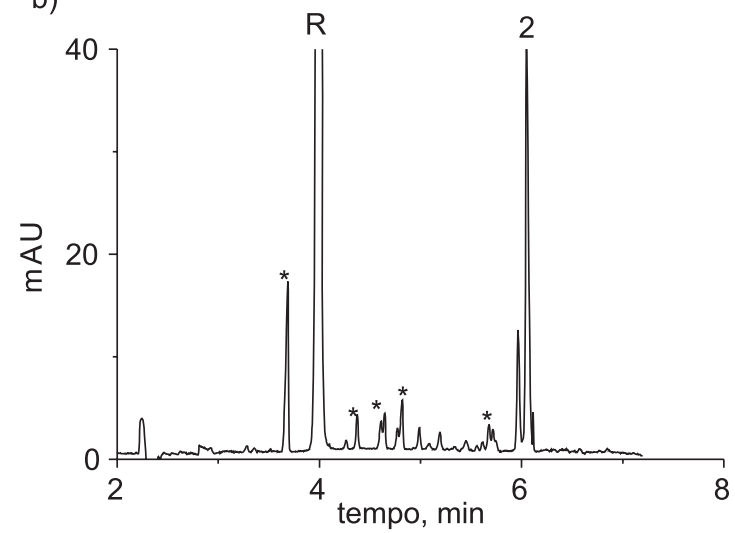

Figura 4. Eletroferogramas de amostras de álcool combustível para inspeção de aldeídos. a) Amostra 1, b) Amostra 2. Eletrólito: $40 \mathrm{mmol} L^{-1}$ de SDS contendo $20 \mathrm{mmol} L^{-1}$ de tetraborato de sódio e $12 \mathrm{mmol} \mathrm{L}^{-1} \mathrm{de} \beta$-CD, $5 \mathrm{~s} x$ $2500 \mathrm{~Pa}$; tensão: $20 \mathrm{kV}$; temperatura: $27^{\circ} \mathrm{C}$; detecção em $216 \mathrm{~nm} . \quad R$. Reagente, 2. Acetaldeído, 3. Acetona, $e^{*}$ não identificado
Tabela 8. Resultados das determinações de acetaldeído e acetona em amostras de álcool combustível

\begin{tabular}{ccc}
\hline Amostras & Acetaldeído ${ }^{\mathrm{a}}\left(\mathrm{mg} \mathrm{L}^{-1}\right)$ & Acetona $^{\mathrm{a}}\left(\mathrm{mg} \mathrm{L}^{-1}\right)$ \\
\hline Amostra 01 & $77,2 \pm 1,2$ & $78,9 \pm 1,5$ \\
Amostra 02 & $72,1 \pm 1,1$ & $\mathrm{ND}$ \\
Amostra 03 & $51,3 \pm 0,8$ & $\mathrm{ND}$ \\
Amostra 04 & $32,0 \pm 0,5$ & $91,3 \pm 1,8$ \\
Amostra 05 & $77,8 \pm 1,5$ & $93,7 \pm 1,9$ \\
Amostra 06 & $35,6 \pm 0,5$ & $\mathrm{ND}$ \\
Amostra 07 & $27,9 \pm 0,4$ & $\mathrm{ND}$ \\
\hline
\end{tabular}

a duplicata; ND não detectado

\section{CONCLUSÃO}

O eletrólito de corrida contendo simplesmente nitrato de sódio e ácido monocloroacético mostrou grande potencial para separação e quantificação de cloreto e sulfato. Com relação à introdução da amostra na coluna capilar, o modo hidrodinâmico apresentou melhores resultados. $\mathrm{O}$ método desenvolvido apresentou bons resultados no que diz respeito à sensibilidade, precisão, recuperação e tempo de análise sendo, portanto, perfeitamente aplicável à amostras de álcool combustível.

O procedimento analítico desenvolvido para análise de aldeídos tem como características a simplicidade e rapidez. O reagente MBTH, utilizado para derivação dos aldeídos, não requer purificação e é de fácil preparação. A separação do acetaldeído dos seus isômeros de posição foi possível com a adição de $\beta$-CD ao eletrólito de corrida. Com base nos resultados apresentados neste trabalho, a metodologia proposta pode ser considerada uma boa alternativa para análise de impurezas orgânicas em amostras de álcool combustível.

\section{AGRADECIMENTOS}

Ao Conselho Nacional de Desenvolvimento Científico (CNPq) e a Fundação de Amparo à Pesquisa do Estado de São Paulo 
(FAPESP) pelas bolsas de produtividade (301201/94-3, 300520/ 92) e pós-doutorado (02/10197-1), respectivamente.

A. A. Cardoso agradece à Financiadora de Estudos e Projetos (FINEP) e Agência Nacional de Petróleo (ANP) pelo suporte financeiro.

\section{REFERÊNCIAS}

1. http://www.ipef.br/listas/bioenergia-I/aug2000/msg00000.html, acessada em Agosto 2004.

2. http://www.unica.com.br, acessada em Agosto 2004.

3. Bishop, G. A.; Stedaman, D. H.; Environ. Sci. Technol. 1990, 24, 843.

4. Rosillo-Calle, F.; Cortez, L. A.; Biomass and Bioenergy 1998, 14, 115.

5. http://www.portalunica.com.br/referencia/estatisticas.jsp, acessada em Janeiro 2004.

6. http://www.anp.gov.br, acessada em Janeiro 2004

7. Seinfeld, J.H.; Pandis, S. N.; Atmospheric Chemistry and Physics: From Air Pollution to Climate Change, Wiley: New York, 1998.

8. Willians, W. J.; Handbook of Anion Determination, Butterworths: London, 1979.

9. AOAC International; Official Methods of Analysis of the Association of Official Analytical Chemists, 12 ${ }^{\text {th }}$ ed., AOAC: Washington, 1975.

10. Vairavamurthy, A.; Roberts, J. M.; Newman, L.; Atmos. Environ. 1992, 26 A, 1965.

11. Vogel, M.; Büldt, A.; Karst, U.; Fresenius J. Anal. Chem. 2000, 366, 781.

12. Bryan, R. J.; Hyde, D. L.; Levaggi, D. A.; Locke, D. C.; Rasmussen, R. A.; Methods for Air Sampling and Analysis, $3^{\text {rd }}$ ed., Lewis: New York, 1989.

13. Tavares, M. F. M.; Quim. Nova 1997, 20, 493.
14. Munoz, R. A. A.; Richter, E. M.; de Jesus, D. P.; Lago, C. L.; Angnes, L.; J. Braz. Chem. Soc. 2004, 15, 523.

15. Colombara, R.; Tavares, M. F. M.; Massaro, S.; Quim. Nova 1997, $20,512$.

16. Pereira, E. A.; Tavares, M. F. M.; Jager, A. V.; Silva, C. L.; Moraes, E. P.; Lima, E. C.; Fonseca, F. N.; Tonin, F. G.; Micke, G.; Santos, M. R.; Oliveira, M. A. L.; Moraes, M. L.; Kampen, M. H.; Fujiya, N. M.; J. Braz. Chem. Soc. 2003, 14, 281.

17. Pereira, E. A.; Tese de Doutorado, Universidade de São Paulo, Brasil, 2002.

18. Ribani, M.; Bottoli, C. B. G.; Collins, C. H.; Jardim, I. C. S.; Melo, L. F. C.; Quim. Nova 2004, 27, 771.

19. Garfield, F. M.; Quality Assurance Principles for Analytical Laboratories, AOAC International: Arlington, 1991.

20. Baker, D. R.; Capillary Electrophoresis, Wiley: New York, 1995.

21. Pereira, E. A.; Stevanato, A.; Cardoso, A. A.; Tavares, M. F. M.; Anal. Bioanal. Chem. 2004, 380, 178.

22. Haddad, P. R.; Doble, P.; J. Chromatogr., A 1999, 834, 189.

23. Thornton, M. J.; Fritz, J.; J. Chromatogr., A 1997, 770, 301.

24. Nielen, M. W. F.; J. Chromatogr., A 1991, 588, 32.

25. Weinberger, R.; Practical Capillary Electrophoresis, Academic Press: New York, 1996.

26. McLaughlin, G. M.; Weston, A.; Hauffe, K. D.; J. Chromatogr., A 1996, $744,123$.

27. Tavares, M. F. M.; Quim. Nova 1996, 19, 173.

28. AOAC Peer-Verified Methods Program; Manual on Policies and Procedures, AOAC: Arlington, 1993.

29. Pereira, E. A.; Cardoso, A. A.; Tavares, M. F. M.; Electrophoresis 2003, 24, 700 .

30. Takeda, S.; Wakida, S.; Yamane, M.; Higashi, K.; Electrophoresis 1994, 15, 1332.

31. Swartz, M. E.; Krull, I.; Pharm. Technol. 1998, $2,4$. 